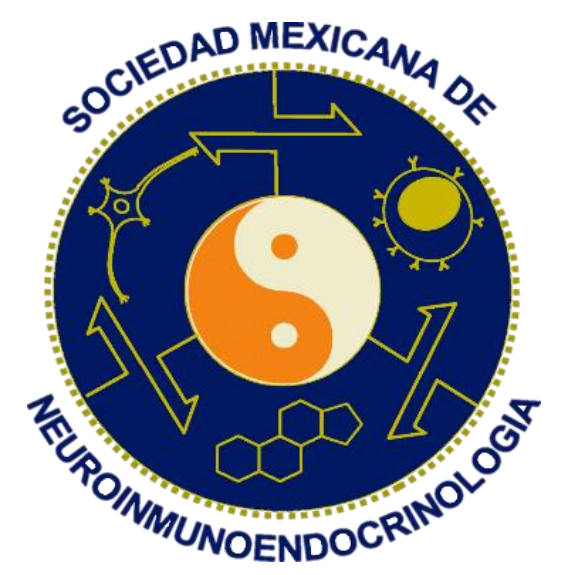

\title{
Simposio: Dimorfismo
}

\section{sexual, salud y enfermedad.}

IV Congreso de la Sociedad Mexicana de Neuroinmunoendocrinología.

Puerto Vallarta Jalisco, México. 21 al 24 de Octubre del 2019.

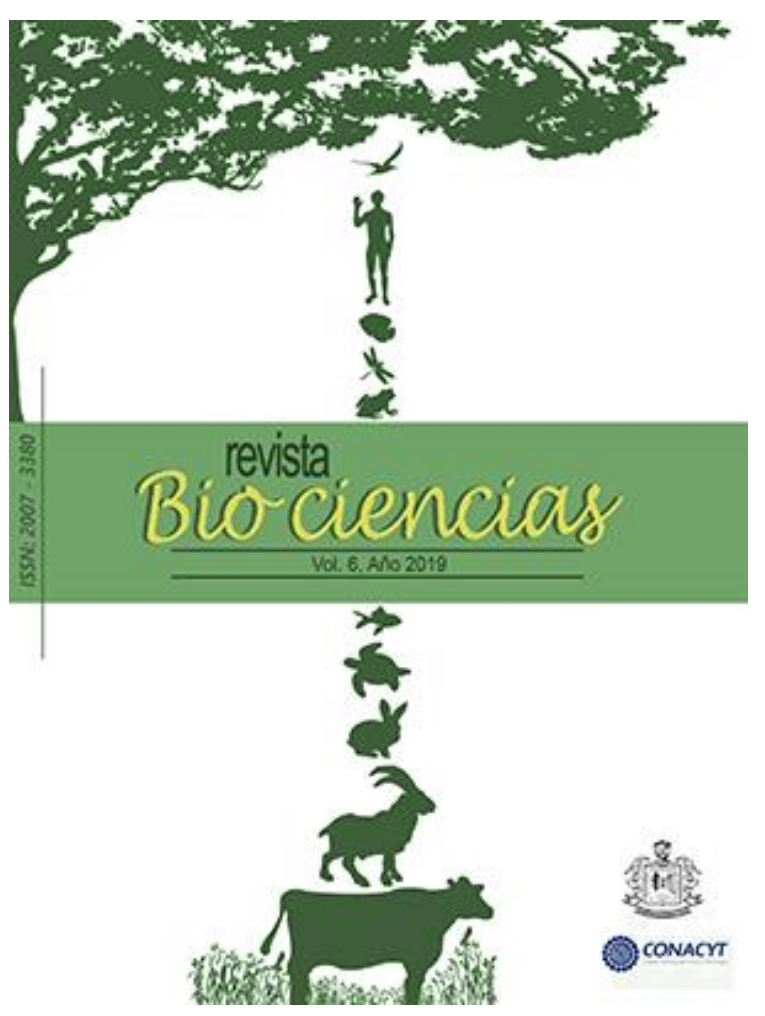




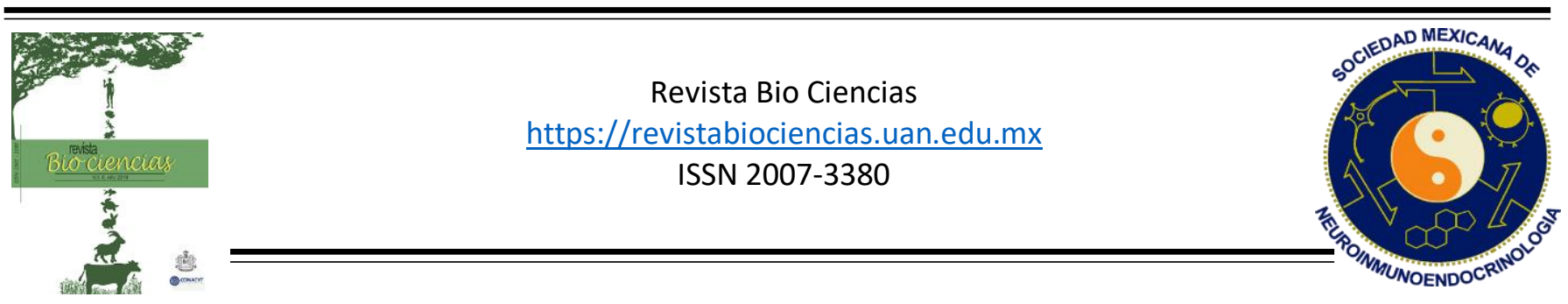

Simposio: Dimorfismo sexual, salud y enfermedad.

\title{
Ligandos metabólicos como activadores del sistema inmune: bases moleculares del inmunometabolismo.
}

\author{
Manjarréz-Reyna A.N. ${ }^{1}$, Martínez-Reyes C.P. ${ }^{1}$, Méndez-García L.A. ${ }^{1}$, Grün J.L. ${ }^{2}$, Escobedo G. ${ }^{1}$ \\ ${ }^{1}$ Laboratorio de Proteómica, Dirección de Investigación, Hospital General de México "Dr. Eduardo \\ Liceaga", 06720, Ciudad de México, México. ${ }^{2}$ Department of Surgery, University Medical Centre \\ Mannheim, Medical Faculty Mannheim, Heidelberg University, D-68167, Mannheim, Germany.
}

La disfunción metabólica es un estado patológico caracterizado por alteración en el metabolismo de lípidos, proteínas y carbohidratos, que se asocia con el riesgo de desarrollar enfermedades como Diabetes Mellitus tipo 2 (DM2), enfermedad del hígado graso no alcohólico y enfermedades cardiovasculares. La disfunción metabólica está íntimamente ligada a la inflamación sistémica de grado bajo, en donde moléculas como la glucosa, el colesterol LDL y el ácido úrico funcionan como activadores o ligandos metabólicos para monocitos y macrófagos. De acuerdo con la expresión de CD14 y CD16, los monocitos humanos se dividen en clásicos, intermedios y no clásicos, éstos últimos con capacidades proinflamatorias importantes a través de la producción de interleucina (IL) 1 beta. Los macrófagos pueden activarse de forma clásica (M1) o alternativa (M2), mostrando funciones proinflamatorias o antiinflamatorias antagónicas, respectivamente. En nuestro grupo de trabajo hemos observado que pacientes con DM2 de reciente diagnóstico muestran una elevación significativa en la cantidad de monocitos proinflamatorios acompañada de niveles circulantes bajos de IL-10, en comparación con sujetos normoglicémicos. Nuestros estudios in vitro confirman que una concentración de $15 \mathrm{mM}$ de glucosa induce la expresión de CD11c, la sintasa de óxido nítrico inducible (NOS-2) y el factor de necrosis tumoral alfa (TNF-alfa), tres marcadores proinflamatorios típicos, en macrófagos humanos. Por otro lado, esta concentración elevada de glucosa también reguló a la baja la expresión de IL-10 y arginasa 1 (Arg-1) en las mismas células, y el uso de inhibidores específicos demostró que este efecto es mediado a través de la vía sorbitol-ROS-NFKB. De forma similar, el ácido úrico disminuyó la síntesis de CD206 e IL-10 en macrófagos humanos in vitro. Por otro lado, niveles bajos de lipoproteínas de alta densidad (HDL) aumentó la subpoblación de monocitos no clásicos productores de IL-1 beta tanto in vitro como en sujetos con síndrome metabólico. En conjunto, estos resultados demuestran que ligandos metabólicos como la glucosa, el ácido úrico y el HDL funcionan como activadores inmunológicos no prototípicos para monocitos y macrófagos humanos, información que podría contribuir al desarrollo de terapias farmacológicas encaminadas al control de diversas enfermedades cardiometabólicas.

Cite this paper/Como citar este artículo: Manjarréz-Reyna A.N., Martínez-Reyes C.P., Méndez-García L.A., Grün J.L., Escobedo G. (2019). Ligandos metabólicos como activadores del sistema inmune: bases moleculares del inmunometabolismo. Revista Bio Ciencias 6: (Suppl) Memorias IV Congreso de la Sociedad Mexicana de Neuroinmunoendocrinología. e841. http://doi.org/10.15741/revbio.06.Suppl.e841 


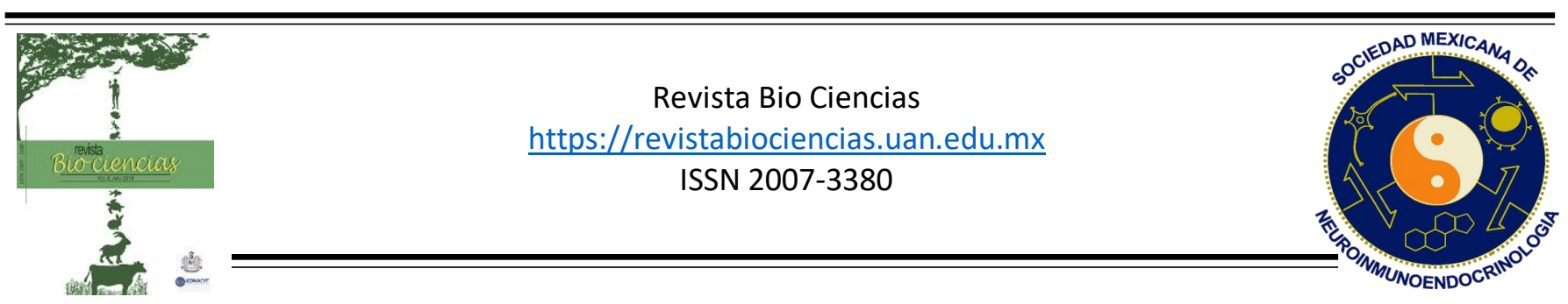

Simposio: Dimorfismo sexual, salud y enfermedad.

\title{
Efecto de los compuestos disruptores endócrinos en la respuesta inmune a la infección por Mycobacterium tuberculosis.
}

\author{
Bobadilla K. \\ Departamento de investigación en inmunologia y medicina ambiental. Unidad de Investigación. \\ Instituto nacional de Enfermedades Respiratorias.
}

Los compuestos disruptores endócrinos (CDEs) son sustancias capaces de alterar el estado de equilibrio hormonal, ya sea mimetizando o antagonizando su acción, provocando un desbalance, no solo hormonal sino también inmunológico. El bisfenol A (BPA) es el compuesto disruptor endócrino más estudiado en los últimos años, este es un compuesto orgánico que se utiliza en la industria manufacturera de policarbonato y resinas epóxicas. Existen otros compuestos orgánicos los hidrocarburos aromáticos policíclicos (HAPs), estos se forman por la combustión incompleta de carbón, combustibles, madera, aceites, petróleo, basura y de materia orgánica en general. Estos y sus nitro-derivados (nitro-HAPs) se han descrito como potenciales mutagénicos e inductores de cáncer. En la fase particulada de la Ciudad de México, los HAPs más abundantes son: benzo[ghi]perileno, indeno[1,2,3-cd]pireno,

benzo[ $b+k]$ fluoranteno, benzo[a]pireno, fenantreno y fluoranteno. Otros compuestos poco estudiados como ftalatos, estos se utilizan como vehículo en plaguicidas, y en la elaboración de productos cosméticos. Los HAPs poseen una estructura que los asemeja a moléculas esteroideas como las hormonas sexuales. Existen diversos estudios in vitro e in vivo en los que se demuestra una actividad tanto estrogénica como anti-estrogénica de compuestos como el benzo[a]pireno y en el caso de los ftalatos, también se ha descrito que son capaces de unirse al receptor de estrógenos inhibiendo su actividad. Dadas estas características estos compuestos pueden funcionar como disruptores endócrinos. Las células del sistema inmune poseen diferentes receptores hormonales, por lo que al ser expuestas a los compuestos disruptores endócrinos pueden afectar su función. El efecto de estos compuestos ha sido poco estudiado en la respuesta inmune por lo que nosotros evaluamos el efecto de estos en la fagocitosis, el procesamiento y presentación de Mycobacterium tuberculosis (Mtb), así como la expresión de receptores hormonales en las células del sistema inmune.Nosotros encontramos una disminución en la fagocitosis y en presentación de Mtb en macrófagos humanos estimulados con Ftalatos e HAPs. Tanto la fagocitosis como la presentación antigénica son procesos fundamentales en el establecimiento de una adecuada respuesta inmune celular protectora a infecciones.

Cite this paper/Como citar este artículo: Bobadilla K. (2019). Efecto de los compuestos disruptores endócrinos en la respuesta inmune a la infección por Mycobacterium tuberculosis. Revista Bio Ciencias 6: (Suppl) Memorias IV Congreso de la Sociedad Mexicana de Neuroinmunoendocrinología. e841. http://doi.org/10.15741/revbio.06.Suppl.e841 


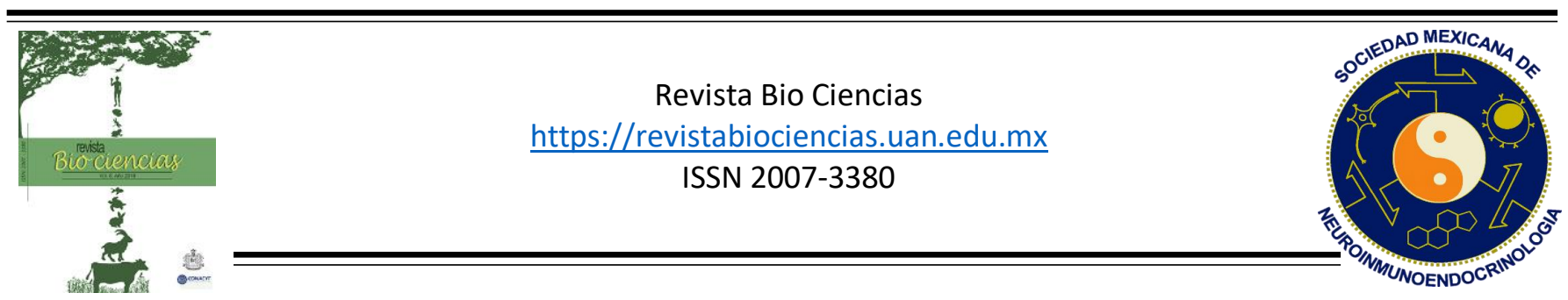

Simposio: Dimorfismo sexual, salud y enfermedad.

\title{
Immunoregulation of experimental autoimmune encephalomyelitis by
}

\section{helminth/derived products.}

\author{
Terrazas L. I. ${ }^{1}$, Peón A. N. ${ }^{2}$ \\ ${ }^{1}$ Unidad de Biomedicina, Facultad de Estudios Superiores-Iztacala, UNAM. ${ }^{2}$ Universidad Autónoma \\ de Hidalgo. E-mail: literrazas@unam.mx.
}

A negative correlation between the geographical distribution of autoimmune diseases and helminth infections has been largely associated with a possible role for such type of infections in the regulation of inflammatory diseases, suggesting new pathways for drug development. However, few helminth-derived immunomodulators have been tested in experimental autoimmune encephalomyelitis (EAE), an animal model for human multiple sclerosis. Here we sought for immunomodulatory activities of Taenia crassiceps excreted/secreted products (TcES) that may suppress EAE development. Interestingly, we found that TCES was able to induce a remarkable suppression on the main signs of EAE, even better than dexamethasone. TCES treatment was still effective at disease dampening even when inoculated at later stages after the onset of EAE. TcES treatment was able to induce a range of Th2-type cytokines, while suppressing both, Th1 and Th17 responses. Also, the polyclonal and the antigen specific proliferative responses of lymphocytes were inhibited in EAE-ill mice receiving TcES, in association with a potent recruitment of suppressor cell populations. Peritoneal inoculation of TcES appears to direct the normal inflammatory cell traffic to the site of injection thus avoiding CNS infiltration, which may work along with Th2 immune polarization and lymphocyte-activation impairment to down-regulate EAE development.

Cite this paper/Como citar este artículo: Terrazas L. I., Peón A. N. (2019). Immunoregulation of experimental autoimmune encephalomyelitis by helminth/derived products. Revista Bio Ciencias 6: (Suppl) Memorias IV Congreso de la Sociedada Mexicana de Neuroinmunoendocrinología. e841. http://doi.org/10.15741/revbio.06.Suppl.e841 